\title{
Influences of domain knowledge on segmentation and memory
}

\author{
Kimberly M. Newberry ${ }^{1}$ - Daniel P. Feller ${ }^{2} \cdot$ Heather R. Bailey $^{3}$ \\ Accepted: 12 November 2020 / Published online: 7 January 2021 \\ (C) The Psychonomic Society, Inc. 2021
}

\begin{abstract}
Much research has shown that experts possess superior memory in their domain of expertise. This memory benefit has been proposed to be the result of various encoding mechanisms, such as chunking and differentiation. Another potential encoding mechanism that is associated with memory is event segmentation, which is the process by which people parse continuous information into meaningful, discrete units. Previous research has found evidence that segmentation, to some extent, is affected by top-down processing. To date, few studies have investigated the influence of expertise on segmentation, and questions about expertise, segmentation ability, and their impact on memory remain. The goal of the current study was to investigate the influence of expertise on segmentation and memory ability for two different domains: basketball and Overwatch. Participants with high and low knowledge for basketball and with low knowledge for Overwatch viewed and segmented videos at coarse and fine grains, then completed memory tests. Differences in segmentation ability and memory were present between experts and control novices, specifically for the basketball videos; however, experts' segmentation only predicted memory for activities for which knowledge was lacking. Overall, this research suggests that experts' superior memory is not due to their segmentation ability and contributes to a growing body of literature showing evidence supporting conceptual effects on segmentation.
\end{abstract}

Keywords Domain knowledge $\cdot$ Event segmentation $\cdot$ Memory $\cdot$ Expertise

Decades of work on domain knowledge (semantic knowledge for a particular field) have shown that experts possess superior memory for information in their expert field. This memory benefit has been explained by various encoding mechanisms, including chunking (Chase \& Simon, 1973), differentiation, and unitization (Herzmann \& Curran, 2011). Recently, another encoding mechanism has been shown to influence memory for event information: event segmentation (Bailey et al., 2013; Flores, Bailey, Eisenberg, \& Zacks, 2017; Newberry \&

The data presented in this manuscript were previously presented at both the 59th annual meeting of the Psychonomic Society in November of 2018, and the 91st annual meeting of the Midwestern Psychological Association in April of 2019.

Kimberly M. Newberry

knewberr@su.edu

1 Department of Psychology, Shenandoah University, 600 Millwood Ave., Halpin Harrison Hall 117, Winchester, VA 22601, USA

2 Department of Learning Sciences, Georgia State University, Atlanta, GA, USA

3 Department of Psychological Sciences, Kansas State University, Manhattan, KS, USA
Bailey, 2019; Sargent et al., 2013; Zacks, Speer, Vettel, \& Jacoby, 2006).

Event segmentation is an encoding mechanism in which people parse continuous event information into meaningful, discrete units (e.g., Zacks, Speer, Swallow, Braver, \& Reynolds, 2007). How people segment an event influences how they perceive, comprehend, and remember events (for review, see Radvansky \& Zacks, 2014). This process may be influenced by both perceptual and conceptual factors, which suggests prior knowledge may affect how someone perceives and segments an event, which in turn may influence memory. While some studies suggest that domain knowledge influences segmentation (e.g., experts identify fewer boundaries: Bläsing, 2015; experts agree more on coarse boundaries: Levine, Hirsh-Pasek, Pace, \& Michnick Golinkoff, 2017; Zacks \& Tversky, 2003), several questions remain: To what extent do people agree on how activities are segmented within and outside of their knowledge domain? Do high-domainknowledge individuals organize events at encoding differently from low-domain-knowledge individuals? If so, does this explain the observed memory benefit?

Thus, the current study investigated the influence of domain knowledge on segmentation and memory of basketball and Overwatch games. These activities were chosen for their popularity as well as for testing the generalizability of 
knowledge effects on segmentation across different activities. To begin, theories of event cognition, event segmentation theory and the event horizon model, are discussed, followed by the relationship between segmentation and knowledge. Afterwards, the literature on expertise is described and integrated with event segmentation, and general predictions about the current study are presented.

\section{Event segmentation theory}

According to event segmentation theory (EST; Kurby \& Zacks, 2008; Zacks et al., 2007), events are experienced continuously, but perception of those events is not. Rather, people use perceptual (e.g., motion, body position; Newtson, Enquist, \& Bois, 1977; Zacks, 2004) and conceptual (e.g., knowledge, goals; Levine et al., 2017; Radvansky \& Zacks, 2014; Zacks, 2004) information to construct mental representations of ongoing activity, such that the current event representation is held in working memory until a change is perceived, at which point a new representation is constructed to reflect the new event (e.g., Zacks et al., 2007). This updating process is thought to occur when there is a mismatch between expectation and reality (Rescorla \& Wagner, 1972) that is driven by prediction failures (Zacks et al., 2007), a lack of coherence (Gernsbacher, 1991), or changes in context (Clewett \& Davachi, 2017).

EST posits that people generate predictions for upcoming actions, and the accuracy of these predictions is monitored. For example, after a basketball player makes a shot, it is likely that a player from the opposite team will inbound the ball and dribble it to the other end of the court. However, when the player with the ball reaches the opposite end of the court, the event becomes less predictable. Will the player pass the ball or take a shot? The points in time when predictions fail, or when people perceive a change and update their event representation, are called event boundaries. Research suggests that within an event, predictability is high, but across event boundaries, predictability is low (e.g., Reynolds, Zacks, \& Braver, 2007; Zacks, Kurby, Eisenberg, \& Haroutunian, 2011). Interestingly, people reliably parse events at consistent boundaries (e.g., Bower, Black, \& Turner, 1979; Hard, Tversky, \& Lang, 2006b; Newtson, 1973; Speer, Swallow, \& Zacks, 2003; Zacks, Tversky, \& Iyer, 2001a), even up to 1 year later (test-retest; Speer et al., 2003).

Research using a unitization paradigm, in which people denote boundaries while watching events unfold, suggests that events are hierarchically structured (e.g., Newtson, 1973; Sargent et al., 2013; Zacks, Tversky, et al., 2001a) such that larger, coarse-grain events are made up of smaller, fine-grain events (Tversky, Zacks, \& Martin, 2008; Zacks \& Swallow, 2007; Zacks, Tversky, et al., 2001a). For instance, a college basketball game may consist of the first half and the second half. However, the first half could be further divided into smaller subevents, such as a series of plays executed by each team. Previous work has found individual differences in the extent to which people perceive alignment between fine and coarse-grain events (e.g., Hard, Lozano, \& Tversky, 2006a; Kurby \& Zacks, 2011; Sargent et al., 2013; Zacks et al., 2001b), and evidence suggests that hierarchical encoding may be important for memory (Kurby \& Zacks, 2011).

Importantly, the event horizon model (Radvansky, 2012), which subsumes event segmentation theory (e.g., Radvansky \& Zacks, 2014, 2017), explains that event boundaries reduce retroactive interference by separating information into separate event models, which leads to better overall memory for the activity. Indeed, evidence suggests that the extent to which people demonstrate normative segmentation (i.e., the degree to which they agree on locations of event boundaries and have better hierarchical alignment) predicts how well they later remember the activity (Bailey et al., 2013; Flores et al., 2017; Kurby \& Zacks, 2011; McGatlin, Newberry, \& Bailey, 2018; Newberry \& Bailey, 2019; Sargent et al., 2013; Zacks et al., 2006).

\section{What influences segmentation behavior?}

Two types of factors presumably influence segmentation: perceptual and conceptual (e.g., Zacks, 2004; Zacks et al., 2007). Much of the research on segmentation has focused on the influence of perceptual cues. For example, perceived event boundaries tend to align with changes in body position (Newtson et al., 1977), spatial location (Magliano, Miller, \& Zwaan, 2001), object motion (Zacks et al., 2001b), and perceptual change (Hard et al., 2006b). For example, perceptual change in basketball may involve changes occurring around the ball (e.g., passes, shots; Huff et al., 2017). Further, regions of the brain that process motion (e.g., extrastriate motion complex) show increased activity at event boundaries (Speer et al., 2003; Zacks et al. 2001b), suggesting that motion is a strong predictor of event boundary perception.

In contrast, research investigating effects of conceptual factors on segmentation is mixed: Some studies suggest that conceptual factors have no influence on segmentation (e.g., Hard et al., 2006b; Huff et al., 2017; Zacks, Kumar, Abrams, \& Metha, 2009), whereas others suggest that they do (context: Loschky, Larson, Magliano, \& Smith, 2015; Newberry \& Bailey, 2019; familiarity: McGatlin et al., 2018; Smith, Newberry \& Bailey, 2020; Zacks \& Tversky, 2003; perspective: Newberry \& Bailey, 2019; schema and scripts: Bartlett, 1932; McGatlin et al., 2018; Schank \& Abelson, 1977; goals: Baldwin, Baird, Saylor \& Clark; 2001; Wilder, 1978a, 1978b; Zacks, 2004). For example, Wilder (1978a, 1978b) showed that participants segmented more often when an actor's goals were unclear as compared with when the activity was goal- 
directed and predictable, indicating that goals affect how people perceive an activity. Similarly, Zacks (2004) found that movement predicted segmentation less when events were goal-directed as opposed to random. Though altogether these results suggest that when goal-related knowledge is present, people rely less on perceptual cues while perceiving an event, the effects have been moderate to small.

A stronger manipulation: Expertise Recent research on knowledge and segmentation has moved toward using a stronger manipulation of prior knowledge: expertise (e.g., Bläsing, 2015; Levine et al., 2017). The use of expertise to evaluate knowledge effects on segmentation fits well with EST and the event horizon model because ample evidence suggests that having prior knowledge about an activity improves prediction when viewing similar activities (e.g., Ambrosini et al., 2013; Kanakogi \& Itakura, 2011; Möller, Zimmer, \& Aschersleben, 2015; Sommerville, Woodward, \& Needham, 2005), and research has shown that people with prior knowledge or experience for an activity also have better memory for that activity (e.g., basketball: Allard, Graham, \& Parsalu, 1980; dance: Allard \& Starkes, 1991; chess: Chase \& Simon, 1973; baseball: Chiesi, Spilich, \& Voss, 1979; bridge: Engle \& Bukstel, 1978; maps: Gilhooly, Wood, Kinnear, \& Green, 1988; music: Meinz \& Salthouse, 1998). Given that prediction is posited to be the mechanism upon which segmentation operates (e.g., Zacks, Braver, et al., 2001b; Zacks, Kurby, et al., 2011) and event boundary identification is important for memory (e.g., Radvansky \& Zacks, 2014), this would suggest that segmentation behavior and memory may differ when one has prior knowledge or experience with an activity compared with no knowledge or experience.

Such a presumption has been supported in the expertise literature focusing on other mechanisms involved in perceptual learning (Goldstone, 1998): differentiation (ability to separate initially fused categories) and unitization (ability to integrate individual parts into functional wholes). Evidence suggests that experts better judge when to engage in each process (Herzmann \& Curran, 2011). When encoding dynamic activity, experts may be better at identifying conceptual units of information and distinguishing between fine details for events within their domain (e.g., Piras, Lobietti, \& Squatrito, 2010). For example, a basketball expert may be able to identify the steps involved in a pick and roll (i.e., better differentiation) whereas a novice might perceive these steps as one action or not at all, or the basketball expert may perceive that same pick and roll as part of a larger play, whereas the novice may perceive it as its own event (i.e., better unitization). If experts identify meaningful event boundaries based on a shared knowledgebase that improves their prediction accuracy, one might expect experts to show more normative segmentation ability, in terms of higher agreement on event boundary locations and/or better alignment of coarse and fine boundaries. ${ }^{1}$

Two studies have investigated effects of expertise on segmentation behavior. In the dance domain, Bläsing (2015) investigated effects of expertise and movement-specific familiarity on the segmentation of a dance phrase. Dancers and nondancers watched and segmented videos of a dancer completing a choreographed phrase. Bläsing (2015) found that dancers segmented less often compared with nondancers, suggesting that expertise reduces the number of perceived boundaries for events within one's area of expertise. In another experiment, Bläsing evaluated the causal role of knowledge on segmentation by having intermediate dancers segment a dance phrase, then learn and practice the motor movements, and segment the phrase again. Like the first experiment, increased familiarity and motor experience with the dance phrase caused dancers to segment less often. Similarly, Levine et al. (2017) found that figure skating experts identified more similar coarse-grain events compared with novices when segmenting an Olympic figure skating routine. These studies have provided initial evidence that expertise influences segmentation behavior; however, some limitations remain. One limitation is that these studies only evaluated segmentation at one grain size. They either provided no specific grain size instruction (Bläsing, 2015) or they only instructed participants to segment at the coarse-grain level (Levine et al., 2017). By including both coarse and fine-grained segmentation in one study, we can evaluate the hierarchical alignment of small events into larger events, and whether domain knowledge increases this alignment. Critically, neither study investigated experts' segmentation ability in a domain outside their expertise. Furthermore, neither study measured memory, so effects of domain knowledge and segmentation on memory have not yet been evaluated.

Given that normative segmentation is associated with better memory for events (Bailey et al., 2013; Flores et al., 2017; Zacks et al., 2006), it is possible that the superior memory of experts may be due to more normative segmentation of the activity within their knowledge domain. If segmentation is a process that is enhanced by accumulation of prior knowledge and experience, one might expect the memory benefit to only be present for the more knowledgeable activity. However, prior work has shown that people use prior knowledge to fill in the gaps at retrieval (e.g., Hasher \& Griffin, 1978). Thus, knowledge could override effects of segmentation on memory and some evidence suggests that segmentation and knowledge affect memory independently (Sargent et al., 2013). If this is true, one might expect segmentation to predict memory only for the novice activity, as novices would not have knowledge

\footnotetext{
${ }^{1}$ Segmentation frequency and agreement are different. Someone may segment less often, but still identify several boundaries identified by the group, and thus have high agreement.
} 
to rely on at retrieval, other than the event representations they built while encoding the activity for the first time.

Thus, the current study expanded upon Bläsing (2015) and Levine et al. (2017) by investigating segmentation behavior and its relationship to memory performance in people with high and low knowledge (for simplicity, we heretofore refer to them as "experts" and "control novices," respectively), across two different domains: basketball (sport) and Overwatch (video game). Basketball is a limited-contact, team sport that involves players working together to achieve a common goal (i.e., shooting the ball through the hoop to earn points). Overwatch, though also team-based, is a multiplayer first-person shooter video game developed by Blizzard Entertainment, Inc.@. Basketball and Overwatch were chosen as the activities in this study for two reasons. First, the inclusion of two activities makes the current study unique in that experts were tested on activities both within and outside their field of expertise. Second, basketball and Overwatch are different from dance and figure skating (e.g., Ericsson \& Smith, 1991), which allows the research questions to be extended from single-actor to team-based activities.

\section{Hypotheses}

If expertise influences segmentation behavior, then experts should segment less often at the coarse grain (segmentation frequency; Bläsing 2015) and agree more on boundary locations (segmentation agreement; Levine et al., 2017) for activities within their expert field. Alternatively, experts may segment more often, particularly at the fine grain, if they engage in perceptual processes such as differentiation to better distinguish between finer subevents (Piras et al., 2010). We also hypothesized that experts would show greater alignment of coarse and fine boundaries for activities within their expert area (hierarchical alignment). However, if perceptual cues have stronger influence on segmentation than do conceptual factors (Hard et al., 2006b; Huff et al., 2017; Zacks, Speer, \& Reynolds, 2009), then experts and control novices may demonstrate similar segmentation behavior because perceptual cues (motion) are readily available to both groups. Further, we hypothesized that experts would show better memory performance for activities within their field of expertise, based on the significant body of expertise research (for review, see Ericsson \& Smith, 1991; Furley \& Wood, 2016).

Prior work suggests that normative segmentation is associated with better memory for events (e.g., Bailey et al., 2013). Thus, we hypothesized that segmentation ability would predict memory performance, regardless of activity or domain knowledge, such that those with better segmentation agreement and/or hierarchical alignment would have better memory. However, we also predicted that the relationship between segmentation and memory would be stronger in the expert activity if domain knowledge improves memory by enhancing segmentation. Alternatively, some work suggests that general knowledge may influence memory independently of segmentation (Sargent et al., 2013), such that people may rely on knowledge (e.g., schemas, scripts, expectations), when it is available, to help them remember the activity, as opposed to how they encode (segment) that particular instance of that activity. In this case, knowledge may override the relationship between segmentation and memory, such that experts who segment well and those who segment poorly remember similar amounts of information.

\section{The current study}

The purpose of this experiment was to investigate the relationship between domain knowledge, segmentation ability, and memory for events within and outside of one's knowledge area. Previous work has observed effects of expertise on the segmentation of dance phrases (Bläsing, 2015) and a figure skating routine (Levine et al., 2017); however, these studies only evaluated experts' segmentation behavior for events within their field of expertise. Additionally, the hierarchical alignment of different segmentation grains and its effects on memory have yet to be evaluated in this context. In the current experiment, basketball and Overwatch experts and control novices viewed and segmented videos of basketball and Overwatch. Due to recruitment issues, only a very small sample of Overwatch experts participated in the study (see Method section). The current experiment ultimately focused on a within-subjects comparison of basketball experts' segmentation and memory for basketball (area of expertise) and for Overwatch (area outside of expertise) videos as well as a between-subjects comparison of segmentation and memory for basketball activities between basketball experts and control novices.

\section{Method}

Participants A total of 165 participants (see Table 1) were recruited from Kansas State University (KSU). Participants were recruited from psychology courses and from other

Table 1 Participant characteristics $(N=165)$

\begin{tabular}{llll}
\hline Group & $N$ & $\%$ Female & $M_{\text {age }}$ in years $(S D)$ \\
\hline Basketball experts & 35 & 51 & $18.66(.68)$ \\
Control novices & 61 & 90 & $18.82(1.37)$ \\
Intermediates & 55 & 73 & $18.82(1.38)$ \\
Overwatch experts & 12 & 0 & $18.75(1.14)$ \\
Uncategorized & 2 & - & - \\
\hline
\end{tabular}


organizations across campus. To increase recruitment of Overwatch experts, the study was advertised through the KSU eSports Club, which promotes professional competition and spectatorship for Overwatch videogame players and fans. Recruitment yielded 35 basketball experts (Overwatch novices), 12 Overwatch experts (three of which were basketball novices, nine of which had "intermediate" or expert basketball scores), 61 control novices (novices in both activities), two uncategorized, and 55 "intermediate" individuals who scored above the novice, but below the expert thresholds in both areas (see Knowledge Surveys, below).

Predictions for the current experiment were based on an "expert" versus "control novice" comparison. Only individuals who met the criteria for expert or control novice were included in the main analyses. Participants who scored in the "intermediate" range for either activity were only included in the exploratory analyses where knowledge was treated as a continuous variable (see Supplemental Materials). Unfortunately, recruitment of Overwatch experts proved difficult, even after targeting Overwatch players from eSports for several months. Therefore, due to the low sample size, the main analyses of the current experiment also exclude this group (though they are included in the exploratory analyses in the Supplemental Materials). Additionally, eight participants' data (two basketball experts, two control novices, two intermediates, and two uncategorized) were lost due to technological issues. Participants were compensated with course credit or entered into a gift card raffle, depending on from where they were recruited.

Because participants were not randomly assigned to groups, all participants completed a series of cognitive measures (processing speed, vocabulary, semantic knowledge, and working memory; see Supplemental Materials for a full description) to assess individual differences that may have otherwise explained possible segmentation and memory effects. Bayes factors were used to test for evidence of the null hypothesis (i.e., no difference between groups; see Table 2). Bayes factors of less than 1 suggested substantial evidence for the null (e.g., Wetzels \& Wagenmakers, 2012), suggesting no differences between groups on these cognitive abilities.

\section{Materials}

Knowledge survey Knowledge surveys were used to identify experts and novices in basketball and Overwatch. The basketball portion of the survey was a modified version of Feller, Schwan, Wiemer, and Magliano (2018; adapted from French $\&$ Thomas, 1987), such that it was reduced to 23 questions to match the Overwatch survey, which was developed for use in the current study. Both the basketball and Overwatch surveys included 23 questions each about general information regarding each activity, as well as seven self-report familiarity and expertise questions. All questions had five answer options, with the fifth option (e) always stating "I don't know." Experts were identified with scores ranging from 17 to 23 , while novices were identified with scores ranging from 0 to 7 (based on percentage cutoffs from previous work using knowledge surveys; Rawson \& van Overschelde, 2008). Both surveys are included in the Appendix.

Videos Five videos were used in this experiment (one practice; four experimental). The practice video depicted a man using Legos to build a ship (155 s). Two of the experimental videos were college basketball games; specifically, Memphis vs. UCLA (153 s; three cuts) and Montana vs. Weber State (130 s; nine cuts; Feller et al., 2018). The other two experimental videos were Overwatch tournament matches; specifically, Houston vs. Boston (144 s; 11 cuts) and London vs. Florida (135 s; seven cuts). All of the experimental videos were shorter clips of continuous game play (maintaining action continuity) taken from longer videos to minimize the influence of cuts on perception, though research suggests that most cuts go unrecognized and do not influence segmentation (Magliano \& Zacks, 2011; T. J. Smith \& Henderson, 2008). Additionally, evidence from the event cognition literature suggests that viewpoint changes also do not influence the events that are perceived (Swallow, Kemp, \& Simsek, 2018). The Overwatch videos were chosen because they were professionally recorded games played by Overwatch experts. Participants viewed all of the experimental videos twice (once per segmentation grain).

Table 2 Performance on cognitive battery by expertise group

\begin{tabular}{lllll}
\hline & Basketball experts & Controls (novices) & $t$ & $p$ \\
\hline Letter comparison & $16.38(.38)$ & $16.08(.34)$ & 0.56 & .58 \\
Pattern comparison & $21.17(.63)$ & $20.28(.60)$ & 0.96 & 0.26 \\
Object naming & $56.12(1.45)$ & $53.44(1.47)$ & 1.20 & .34 \\
Vocabulary knowledge & $14.03(.48)$ & $13.48(.49)$ & 0.73 & .23 \\
R-SPAN & $0.80(.02)$ & $0.77(.02)$ & 1.08 & .47 \\
\hline
\end{tabular}

Note. Standard error of the mean in parentheses. Letter comparison and pattern comparison were both measures of processing speed. Object naming and vocabulary were both measures of semantic knowledge. R-SPAN was a measure of working memory capacity. BF = Bayes factor, evidence for the null. 
Unitization task The unitization task (Newtson, 1973) was used as an overt measure of participants' perception of event boundaries in the videos. While watching the videos, participants were asked to press the space bar each time "one meaningful unit of activity ends and another begins." Participants were instructed to identify larger (coarse) or smaller (fine) units of meaningful activity by pressing the space bar (e.g., Sargent et al., 2013). Participants were shaped on this task using a practice video (see Zacks et al., 2009). The shaping procedure required participants to identify at least 3 larger (coarser) units or 6 smaller (finer) units in order to move on to the experimental trials. If this threshold was not met, participants received feedback stating that other people typically identify more units; however, they were not given explicit examples of how the activities in the video could be segmented. After receiving this message, participants repeated the shaping procedure until they passed the threshold.

\section{Event memory measures}

Recognition Recognition memory was assessed using a two-alternative forced-choice test. There were 20 trials per video, each containing one target and one distractor image, simultaneously presented side-by-side. Target images always came from the videos that participants watched, and distractor images always came from portions of the same video that participants did not see. Presentation order of the image pairs was the same for each participant. Participants received 1 point for each correctly identified image (up to 20 total points). Participants' scores were reported as proportion correct.

Order memory ${ }^{2}$ Order memory was assessed using a twoalternative forced-choice test, based on the measure used by Dubrow and Davachi (2014). For each video, participants were presented with eight image pairs on the computer. All the images came from the videos participants watched. A prompt appeared on screen stating “more recent?", and participants were instructed to choose the image depicting the more recent action.

\section{Design and procedure}

Expertise was a between-subjects variable. Participants $\left(N_{\text {BasketballExperts }}=33, N_{\text {ControlNovices }}=59\right)$ were grouped based on their scores from the knowledge survey about basketball and Overwatch (novice $\leq 7$; expert $\geq 17$; see Table 3; see Supplemental Materials for analyses that

\footnotetext{
${ }^{2}$ Cronbach's alpha $=.22$ indicating that the internal consistency of the order memory task was extremely poor. For these reasons, the memory analyses only include recognition data.
}

Table 3 Knowledge scores by expertise group

\begin{tabular}{lll}
\hline & Basketball experts & Controls (novices) \\
\hline Overwatch & $1.29(.38)$ & $1.34(.28)$ \\
Basketball & $20.06(.27)$ & $4.51(.29)$ \\
\hline
\end{tabular}

Note. Standard error in parentheses. Novice $\leq 7$; Expert $\geq 17$

include expertise as a continuous variable, including participants with intermediate knowledge). To be clear, everyone in the basketball expert group were also novices in Overwatch, separate from those in the control group, who were identified as control novices in both activities. Activity (basketball \& Overwatch) was treated as within subjects, such that all participants viewed and segmented videos of both activities. Participants segmented each video twice: once per grain (coarse vs. fine). Video and distractor task were counterbalanced across participants. Segmentation grain was counterbalanced, such that participants segmented all the videos at one grain, then after completing the last block of tasks for the last video, they segmented all the videos again (in the same order of presentation) at the other grain.

All participants entered the lab in small groups of three or four and were seated at a computer. They first signed an informed consent form and then completed the knowledge survey. After, they were given a demographics form and instructed not to fill it out until the experimental program on the computer told them to do so. Each participant was then presented with the practice video, which shaped each participant's segmentation behavior to whichever segmentation grain order each participant was assigned (i.e., at least three button presses for coarse grain; at least six for fine grain). After completing the shaping procedure, the experimental trials began. The experimental trials consisted of four blocks. In each block, the experimental video was presented, and participants were instructed to "press the space bar any time they felt a meaningful unit of activity ended and a new one began." After each video, participants completed a distractor task (i.e., one of the individual differences measures listed above), and then moved on to the recognition and order memory tasks. Memory task order was not counterbalanced because the viewing of target images in the order memory task could have aided participants on the recognition task. After the order memory task for the last video of the last block, participants were shown the practice video again and trained on the segmentation task for the alternative grain. Participants then resegmented each video at this new grain in the same order in which the videos were originally presented. At the end of the experiment, participants completed the working memory task. Finally, they were debriefed, thanked, and compensated for their time. 


\section{Results}

\section{Data preparation}

Recruitment issues focused the analyses of the current experiment to basketball experts $(N=33)$ and control novices $(N=$ 59; see Participants section, above). Individuals with intermediate knowledge scores and Overwatch experts were included in supplemental exploratory analyses treating knowledge as a continuous variable (see Supplemental Materials). Otherwise, no outliers were identified.

\section{Approach}

The main analyses were conducted using generalized multilevel modeling techniques. These techniques accounted for nonnormal error distributions (e.g., Poisson for count data, logistic for binomial) of the dependent measures, and error variance associated with random effects. Additionally, experimental version (based on activity order and grain order) was not a significant predictor (all $p \mathrm{~s}$ $>$.09). We first evaluated encoding processes (segmentation frequency, agreement, and hierarchical alignment) and then evaluated retrieval processes (recognition). Then, we assessed the extent to which encoding predicted retrieval. Note that we also ran analyses on the full data set $(N=157)$ with knowledge treated as a continuous predictor and replicated the general pattern of results (see Supplemental Materials).
Does domain knowledge affect event encoding?

Unitization Two measures of unitization were used to assess how well people identified and agreed on the locations of boundaries.

Segmentation frequency Segmentation frequency was scored as the total number of button presses (i.e., total number of perceived event boundaries) per video. Bläsing (2015) found that experts identified fewer event boundaries compared with novices; therefore, we predicted a within-subjects difference such that basketball experts would segment less often, regardless of grain, during basketball videos, compared with Overwatch videos. We also expected to find a between-subjects difference such that basketball experts would segment less often compared with the control novices, for the basketball videos. Finally, we expected participants to segment less often at the coarse grain compared with the fine grain, regardless of expert knowledge and activity.

To investigate these hypotheses, a generalized Poisson multilevel model was used to predict segmentation frequency from the full factorial of the fixed effects of group, activity, and segmentation grain, and the random effects of participant and video (see Fig. 1). A significant main effect of grain was present $(z=-49.63, p<.001)$ such that participants identified fewer coarse boundaries $(M=19.51, S E$ $=1.80,95 \% \mathrm{CI}[15.94,23.10])$ than fine boundaries $(M=$ $39.59, S E=2.76,95 \%$ CI $[34.11,45.07])$, regardless of knowledge or activity. The main effects of group and

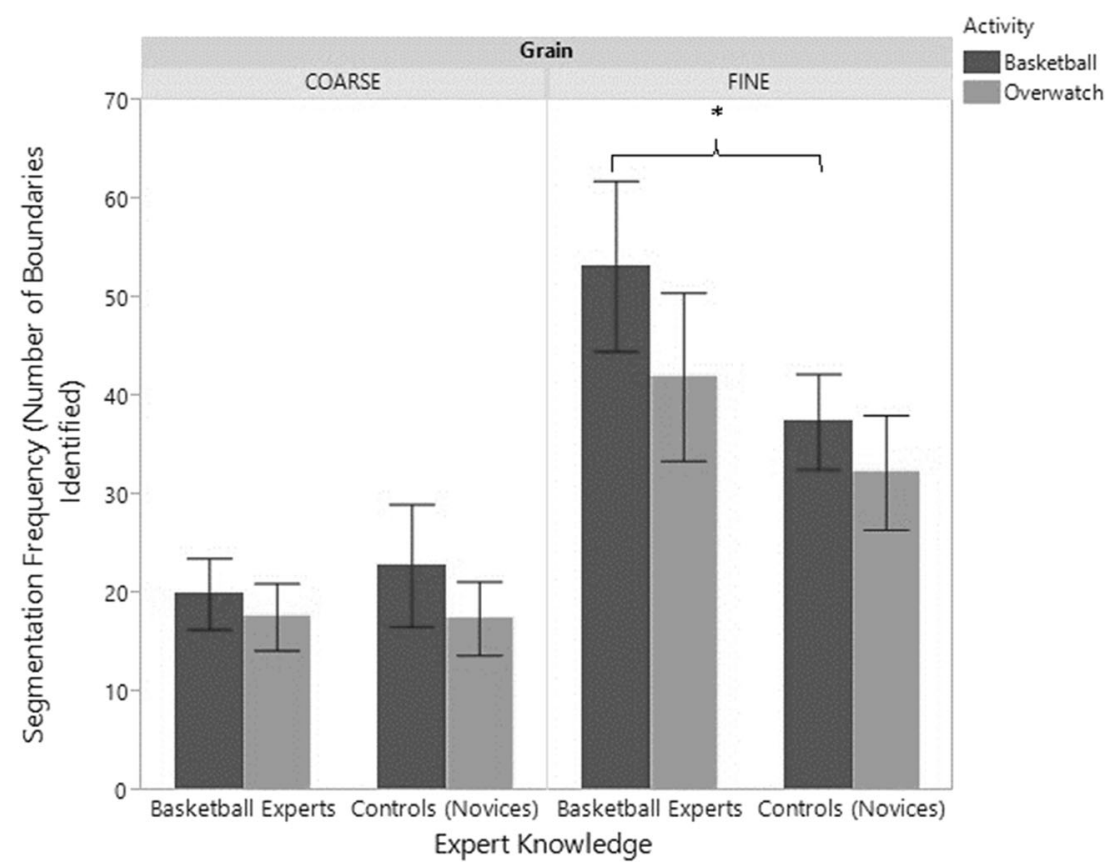

Fig. 1 Number of boundaries identified for each activity, at each grain, by experts and control novices. Error bars indicate standard error of the mean. * is significant .001 
activity were not significant, indicating that there were no baseline differences in the number of perceived events between groups or between activities; however, these fixed effects did interact with grain. A significant two-way interaction between group and grain was present $(z=-11.11, p$ $<.001$ ) such that control novices identified fewer fine boundaries $(M=34.95, S E=3.22,95 \%$ CI [28.50, 41.39]), compared with basketball experts $(M=47.60, S E$ $=4.82,95 \%$ CI $[37.78,57.42]$ ), regardless of activity, but no group differences were present at the coarse grain.

These results were qualified by a significant three-way interaction between group, activity, and segmentation grain $(z=$ $-3.17, p=.002$ ), such that participants identified significantly more fine boundaries than coarse boundaries for the basketball videos, compared with Overwatch, and this difference was greater for basketball experts (basketball coarse: $M=19.95$, $S E=2.42,95 \%$ CI $[15.03,24.88]$; basketball fine: $M=53.20$, $S E=5.55,95 \%$ CI $[41.89,64.51]$; Overwatch coarse: $M=$ 17.61, $S E=2.23,95 \%$ CI $[13.07,22.14]$; Overwatch fine: $M=$ $42.00, S E=5.73,95 \%$ CI $[30.34,53.66])$, compared with control novices (basketball coarse: $M=23.09, S E=3.56$, 95\% CI [15.95, 30.23]; basketball fine: $M=37.43, S E=$ 3.07, 95\% CI [31.28, 43.58]; Overwatch coarse: $M=17.55$, $S E=2.50,95 \%$ CI $[12.55,22.56]$; Overwatch fine: $M=32.17$, $S E=4.00,95 \%$ CI $[24.15,40.20])$. No other effects were present (all $p \mathrm{~s}>.05$ ).

These results did not support the hypothesis that experts would segment less often compared with control novices, particularly for activities in their field of expertise. Instead, results suggest that experts and control novices identified a similar number of coarse boundaries, regardless of activity, and that experts identified more fine boundaries for activities within their field of expertise. This result fits with previous work showing that experts are better able to differentiate between fine details for events within their domain (e.g., Piras et al., 2010).

Segmentation agreement Segmentation agreement refers to how well people agree with others on the locations of perceived event boundaries. Higher segmentation agreement corresponds to more normative segmentation. To calculate agreement, each participant's segmentation data was smoothed by fitting a Gaussian kernel density function around each event boundary (button press), for each video. Each frame of each video received a value ranging from 0 to 1 , indicating the probability or likelihood that the frame was an event boundary. A bandwidth of 25 (i.e., 25 frames per second) was used to correspond to 1-second time bins, such that frames closer to where the participant identified an event boundary received a larger value, compared with frames farther away. Next, the probability associated with each frame or button press was averaged across participants to create normative event boundaries. Finally, each participant's segmentation probability at each frame was correlated with the normative boundaries. ${ }^{3}$ Based on Levine et al. (2017), we predicted a significant between-subjects effect such that basketball experts' segmentation agreement would be higher than control novices for basketball videos, regardless of grain. However, we also expected to observe a within-subjects effect such that basketball experts' segmentation agreement would be higher for basketball videos compared with Overwatch videos, regardless of grain.

To evaluate these hypotheses, a generalized linear multilevel model was used to predict segmentation agreement from the full factorial of the fixed effects of group, activity, and segmentation grain, and the random effects of participant and video (see Fig. 2). A significant main effect of grain was present $(t=-4.05, p<.001)$ such that agreement of fine boundaries $(M=.32, S E=.15,95 \%$ CI $[.28, .34])$ was higher than agreement of coarse boundaries $(M=.26, S E=.01,95 \%$ CI $[.24, .28])$. However, this effect was qualified by a significant three-way interaction between group, activity, and grain $(t=2.29, p=.02)$. All participants showed higher agreement for the basketball videos $(M=.35, S E=.01,95 \%$ CI $[.32, .38])$ compared with the Overwatch videos $(M=.22, S E=.01,95 \%$ CI $[.20, .24])$, but experts $(M=.36, S E=.02,95 \%$ CI $[.30$, .41]) showed a significantly higher agreement compared with the control novices $(M=.29, S E=.02,95 \%$ CI $[.25, .32])$, only at the coarse grain. The within-subjects effect $(d=.96)$ was larger than the between-subjects effect $(d=.42)$. No other effects were significant (all $p \mathrm{~s}>.05$ ).

These results partially support our hypothesis in that experts showed better segmentation agreement, compared with control novices, for activities within their expert domain, but only at the coarse grain. However, recall that experts did not identify significantly more coarse boundaries than control novices (see Fig. 1). Altogether, this suggests that experts' better coarse segmentation agreement was not due to identifying more coarse boundaries, but rather identifying more similar coarse boundaries likely due to their shared knowledge for basketball.

Hierarchical alignment Hierarchical alignment is the extent to which each identified coarse boundary temporally corresponds with an identified fine boundary (Kurby \& Zacks, 2011; Sargent et al., 2013; Zacks et al., 2001a). It is a measure of segmentation organization, or the degree to which each participant's coarse events comprise groups of related fine events (Sargent et al., 2013). One way of measuring hierarchical alignment is by calculating enclosure, which refers to the degree to which groups of related fine events are contained within coarse events (Hard, Recchia, \& Tversky, 2011;

\footnotetext{
${ }^{3}$ Each participant received one correlation score for each of the eight videos. This process was repeated three times, using everyone, domain experts, and one's own group as the different comparison groups for generating the normative boundaries. The main analyses presented used everyone as the comparison (normative) group to increase power.
} 


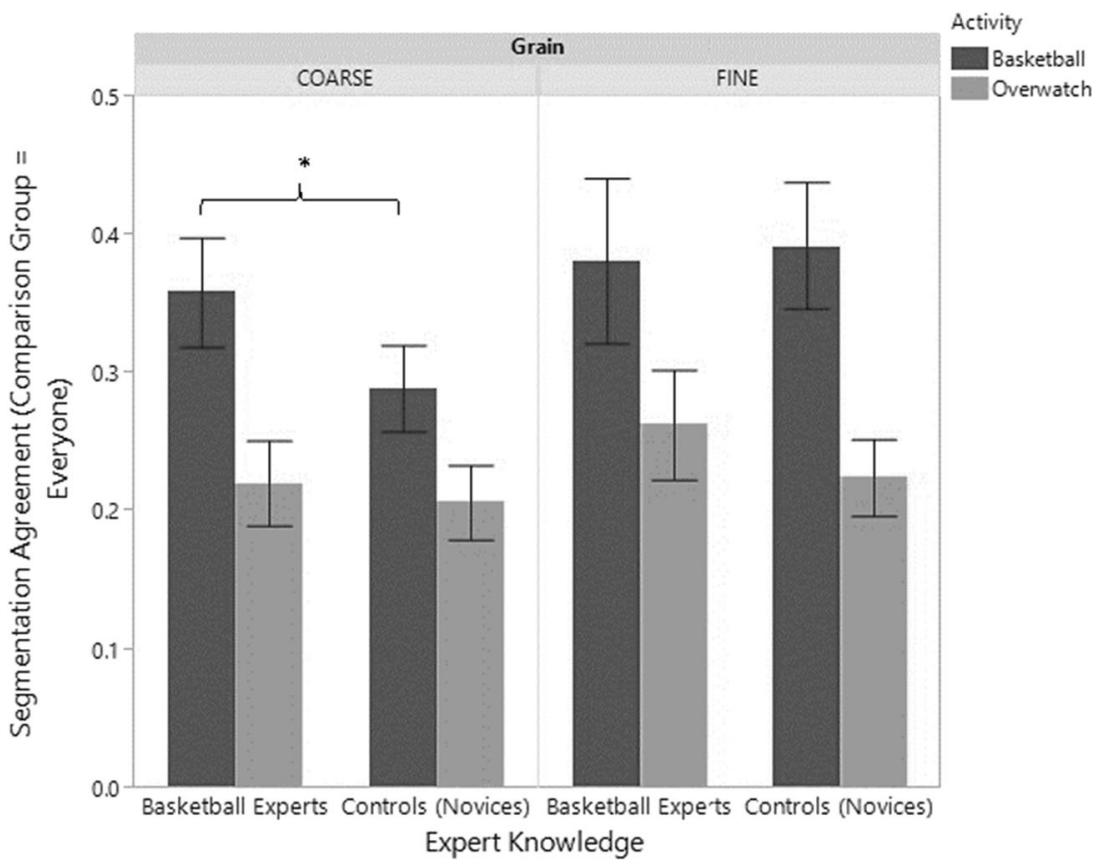

Fig. 2 Segmentation agreement for each activity, at each grain, by experts and control novices. Error bars indicate standard error of the mean. * is significant .05

Sargent et al., 2013). Coarse boundaries were scored based on whether they followed or preceded the closest fine boundary, for each video. Each participant's enclosure score was then the proportion of coarse boundaries that followed (rather than preceded) the closest fine boundary, accounting for expected enclosure due to chance. Higher values indicate better alignment. We predicted a between-subjects effect such that basketball experts, compared with control novices, would exhibit better alignment of coarse and fine boundaries for basketball videos, compared with Overwatch. We also predicted a within-subjects effect such that basketball experts themselves would exhibit better alignment of coarse and fine boundaries for basketball videos, compared with Overwatch, since they themselves were novices in Overwatch.

A generalized linear multilevel model was used to predict enclosure from the fixed effects of group, activity, and their interaction, and the random effects of participant and video. A significant main effect of group $(t=2.07, p=.04)$ and a marginally significant main effect of activity $(t=3.27, p=.07)$ were present; however, these effects were qualified by a significant

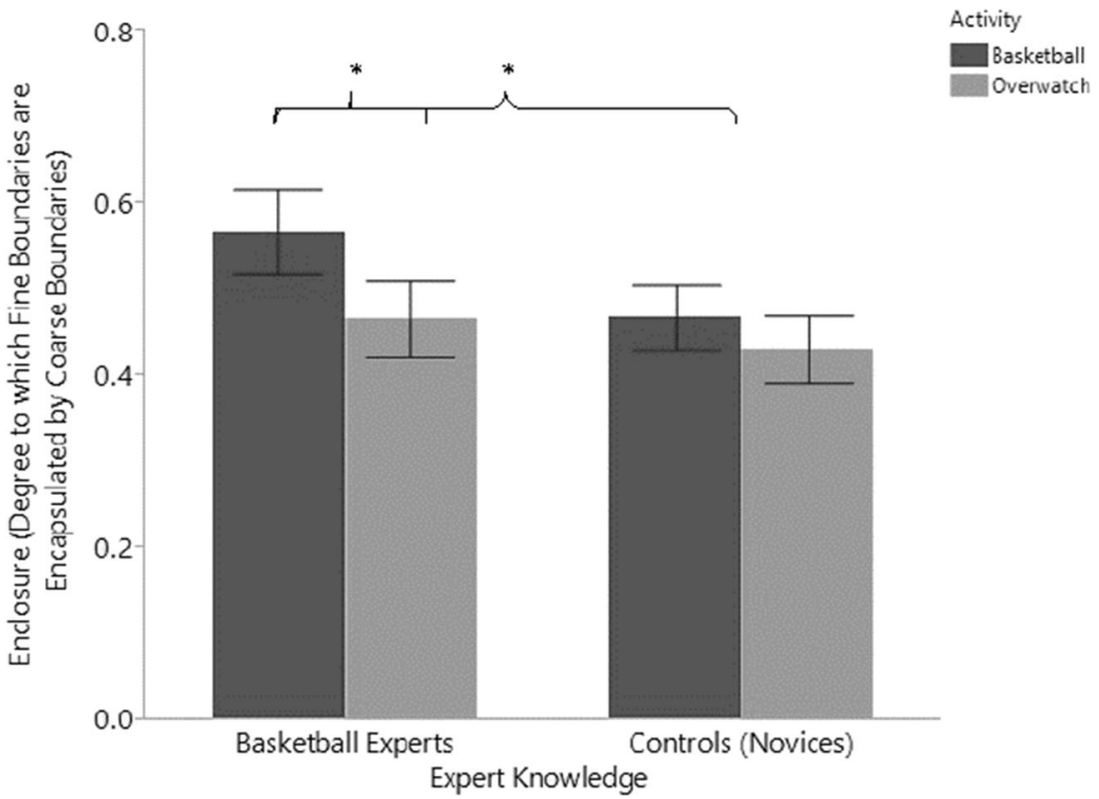

Fig. 3 Enclosure for each activity, by experts and control novices. Similar to temporal distance, scores farther from zero are better. Error bars indicate standard error of the mean. * is significant .05 


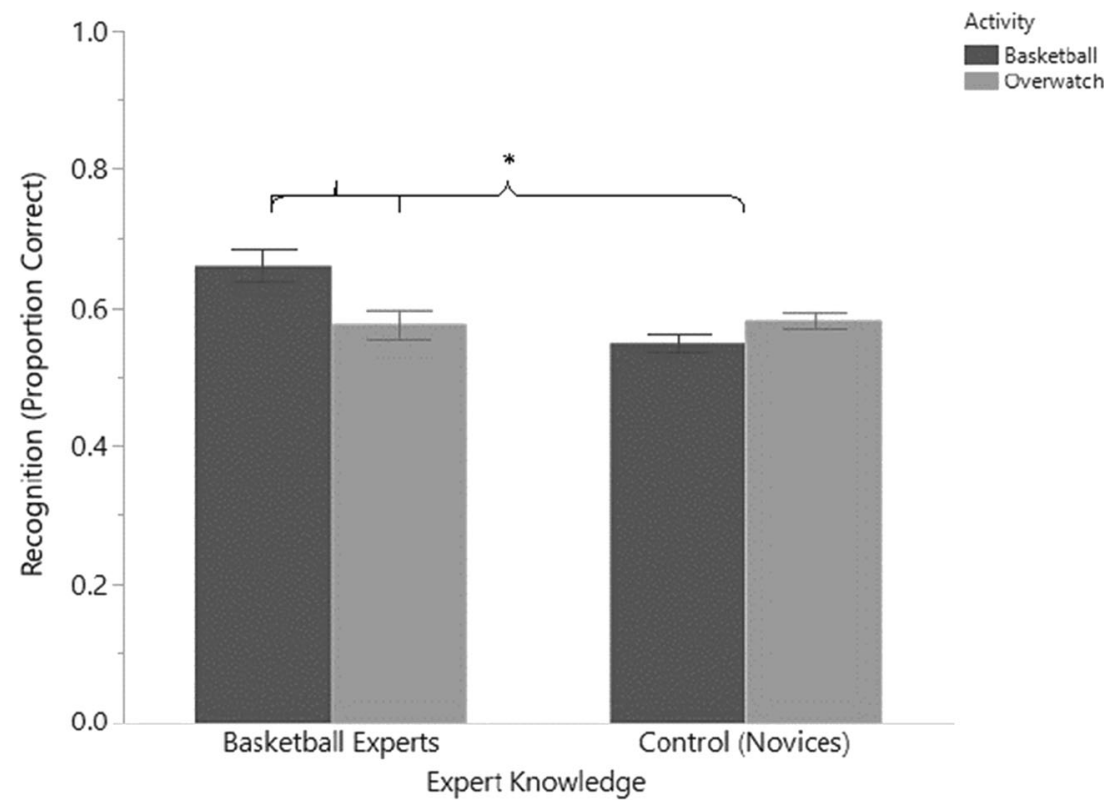

Fig. 4 Recognition performance for each activity, by experts and control novices. Error bars indicate standard error of the mean. *is significant .001

interaction between group and activity $(t=2.03, p=.04)$. Basketball experts exhibited better enclosure for basketball ( $M$ $=.57, S E=.03,95 \% \mathrm{CI}[.50, .63])$, compared with Overwatch ( $M=.47, S E=.03,95 \%$ CI $[.41, .52])$, whereas control novices (basketball: $M=.46, S E=.02,95 \%$ CI [.42, .51]; Overwatch: $M$ $=.43, S E=.02,95 \%$ CI $[.38, .47])$, did not differ in their enclosure ability across the two activities (see Fig. 3). This result supports our hypothesis in that experts showed better encoding organization of activities within their expert domain.

\section{Does expertise affect memory for dynamic activities?}

The majority of work done with experts has shown that experts possess better memory for information within their field of expertise (for review, see Ericsson \& Smith, 1991; Vicente \& Wang, 1998). Based on this, we hypothesized to find a within-subjects effect such that basketball experts would exhibit better recognition memory for basketball videos compared with Overwatch videos. We also hypothesized to find a Group $\times$ Activity interaction such that experts would remember more than control novices for the basketball videos, but they would not differ in their recognition memory performance for the Overwatch videos.

Recognition A generalized logistic multilevel model was used to predict recognition performance from the fixed effects of group, activity, and their interaction, and the random effects of participant and video. A significant interaction between group and activity was present $(z=5.05, p<.001)$ such that basketball experts exhibited significantly better recognition performance for basketball $(M=.68, S E=.02,95 \%$ CI $[.63, .72])$, compared with Overwatch $(M=.59, S E=.02,95 \%$ CI $[.55, .63])$, whereas control novices (basketball: $M=.56, S E=.01,95 \%$ CI $[.53$, $.58]$; Overwatch: $M=.59, S E=.01,95 \%$ CI $[.56, .62]$ ), did not differ in their recognition performance across activities (see Fig. 4). No other effects were present (all $p s>.05$ ). This result supports our expertise hypothesis and replicates the benefit effect of expertise on memory for information in one's expert domain.

\section{Does experts' better segmentation ability explain the memory benefits?}

Segmentation agreement is associated with memory for events (e.g., Bailey et al., 2013; Flores et al., 2017; Sargent et al., 2013); thus, we hypothesized participants with high segmentation ability would also have better memory, regardless of activity. Additionally, we predicted that segmentation agreement would interact with group such that basketball experts would show an even stronger relationship between agreement and memory as compared with the control novices for basketball videos. This prediction is based on the idea that knowledge would improve segmentation agreement, which would in turn improve memory.

Segmentation agreement A generalized Poisson multilevel model was used to predict recognition performance from the full factorial of the fixed effects of segmentation agreement, ${ }^{4}$ group, and activity and the random effects of participant and video. A significant main effect of segmentation agreement $(z=1.96, p=$ .05 ) indicated that recognition was indeed better for people with higher segmentation agreement, replicating prior work (Bailey et al., 2013; Flores et al., 2017; Sargent et al., 2013; Zacks et al., 2006). However, the Segmentation Agreement $\times$ Group

\footnotetext{
${ }^{4}$ We used coarse segmentation agreement here, but fine segmentation agreement produced the same pattern of results.
} 
interaction was not significant, indicating that the effect of segmentation on memory was not stronger for experts.

A significant two-way interaction between knowledge and activity was present $(z=-2.97, p=.003)$, such that basketball experts' recognition was better for basketball videos, compared with Overwatch but control novices' recognition did not differ by activity. A significant two-way interaction between coarse segmentation agreement and activity $(z=-2.23, p=.03)$ indicated that segmentation agreement more strongly predicted recognition for Overwatch than for basketball. However, these two-way interactions were qualified by a three-way interaction between segmentation agreement, group, and activity was marginally significant $(z=1.90, p=.06)$. Segmentation agreement only predicted recognition performance for experts in the Overwatch videos ( $r=$ .38; see Fig. 5). No other effects were present (all $p \mathrm{~s}>.05$ ).

These results partially supported our hypothesis in that segmentation agreement was associated with better memory; however, this relationship was not stronger overall for experts. Experts' segmentation agreement did not explain their improved memory performance in their expert domain. Rather experts' segmentation agreement only predicted their memory performance for the unfamiliar activity, suggesting that segmentation may benefit memory more when people need to rely on encoding efficiency and not semantic knowledge to help remember an activity.

\section{Discussion}

The current study replicated and extended the literature on domain knowledge and event cognition by evaluating whether domain knowledge influences segmentation and memory for events within and outside of one's knowledge field. Overall, basketball experts' segmentation and memory ability for activities within their area of expertise differed from that of the control novices (between-groups comparison) and also differed from their own segmentation and memory ability for the activity outside their area of expertise (within-subjects comparison). Importantly, however, experts' superior memory was not a product of their more normative segmentation ability, suggesting that effects of knowledge and segmentation may influence memory independently. Explanations for these findings are outlined below.

\section{Encoding differences}

Boundary identification Previous work evaluating influences of expertise and familiarity on segmentation has found that fewer subevents are identified as people gain knowledge for, or familiarity with, an activity (e.g., Bläsing, 2015; Hard et al., 2006b; Levine et al., 2017). The current study did not replicate these findings. At the coarse level, basketball experts did not differ from control novices on the number of perceived event boundaries. However, at the fine level, experts identified more event boundaries, particularly for the activity in which they had more knowledge. Neither Bläsing (2015) nor Levine et al. (2017) distinguished between coarse and fine boundary identification.

One possibility is that experts are better at differentiating between information in their expertise area (Herzmann \& Curran, 2011). Evaluation of experts' superior differentiation

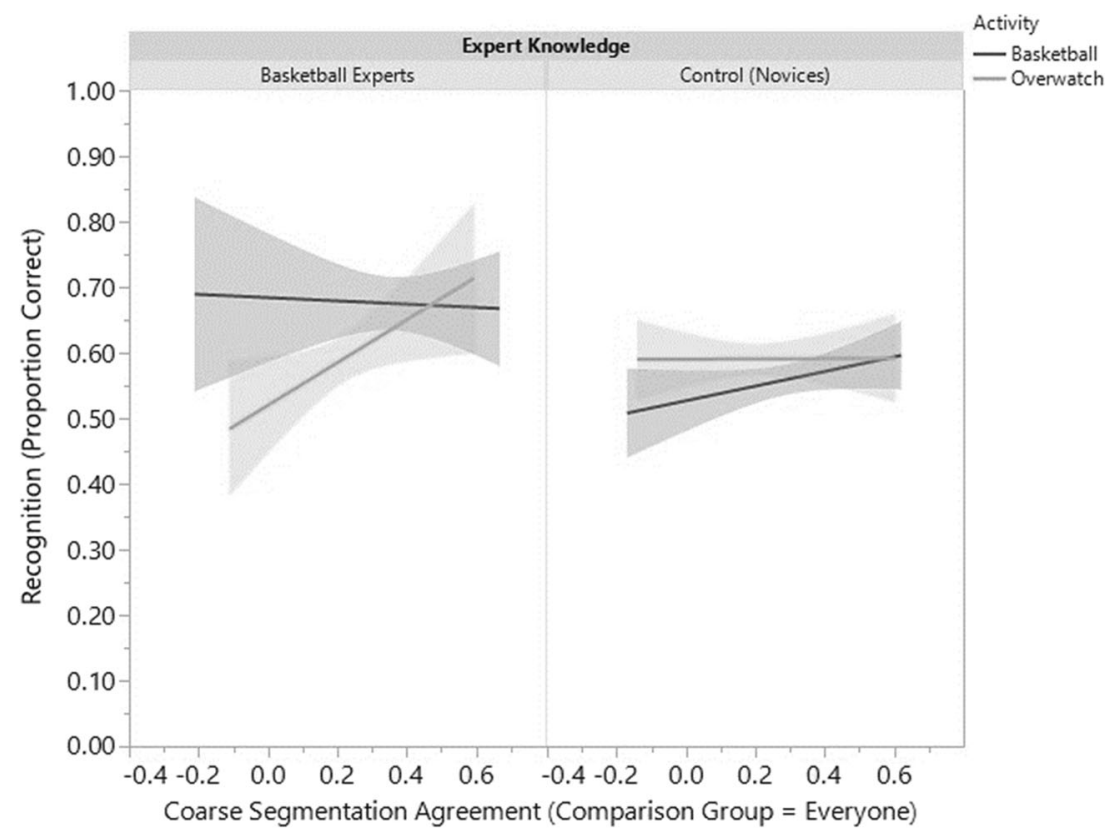

Fig. 5 Coarse segmentation agreement predicts recognition performance. Colored areas indicate confidence of the fit of the line. Shaded areas indicate confidence of the line fit $(95 \%)$ 
abilities has been restricted to object categorization and feature processing, as opposed to perception of dynamic events. Based on the evidence in the current study, experts may engage in differentiation processing when identifying fine subevents for dynamic activities within their field of expertise. Future studies should further evaluate the influence of knowledge on coarse and fine segmentation to better understand how experts perceive event structure within their domain.

Boundary agreement Levine et al. (2017) found that figureskating experts agreed on the major subevents within the figure-skating routine. The current study replicated this effect at the coarse-grain level. Interestingly, this higher agreement among experts at the coarse grain was not due to their identifying more coarse-grain boundaries, because they identified a similar number of coarse boundaries as the control novices. Of the coarse boundaries identified by participants, experts identified more similar boundaries for basketball, whereas control novices displayed more idiosyncratic coarse boundary identification (lower agreement). Experts may use their similar knowledge base to guide their segmentation. Although not explicitly tested in this study, experts may demonstrate greater agreement in their domain because they are better able to track event coherence or experience fewer prediction errors because they can anticipate a wider range of outcomes. It is also possible that experts may be more precise in their timing when identifying boundaries, compared with novices who may be slower to notice important changes. Motor perception research suggests that motor expertise modulates action anticipation (basketball: Aglioti, Cesari, Romani, \& Urgesi, 2008; music: Wöllner \& Cañal-Bruland, 2010), such that observers are better at anticipating actions of others when they themselves have experience performing the same actions.

Interestingly, fine segmentation agreement for experts and control novices did not differ, despite experts identifying more fine boundaries for basketball. It is possible that identification of fine boundaries may be driven by changes in perceptual cues (e.g., motion: when one basketball player passed the ball to another). If experts and novices both rely on motion to guide their segmentation of fine events, they could have identified similar boundaries.

Boundary organization Previous research has found no influence of knowledge on hierarchical alignment differences (Sargent et al., 2013). The current study, however, found experts showed better enclosure of coarse and fine boundaries for basketball. Similarly, Feller et al. (2018) found that basketball experts were better able to perceive structure in basketball games than novices, conceptually replicating the encoding organization effects found with basketball experts in the current study. These studies suggest knowledge influences encoding organization of boundaries; however, future research should continue to investigate effects of event structure on segmentation.
Altogether, experts and control novices differed on most of the dependent measures of encoding, suggesting that experts encode dynamic information within their knowledge field differently than information outside their field. These findings support EST, in that domain knowledge influenced segmentation ability. Importantly, the current findings may not have been present had grain size (coarse and fine) not been included. This manipulation allowed us to investigate the levels of encoding or online event processing on which knowledge may have an effect, which is important for revising EST or translating these effects to applied scenarios (e.g., education).

Retrieval differences The current study replicated decades of research demonstrating experts' superior memory for information within their knowledge field. Basketball experts exhibited more accurate recognition performance compared with control novices, particularly for basketball videos, suggesting that knowledge facilitated memory.

\section{Does encoding predict retrieval? It depends}

A major goal of the current study was to evaluate the extent to which experts' segmentation ability predicted memory within their knowledge field. We found that experts had more knowledge for basketball, and this knowledge was associated with better segmentation ability when encoding parts of a basketball game. The between-subjects effect of knowledge on coarse segmentation was moderate $(d=.42)$ and the within-subjects effect was large $(d=.96)$, thus showing comparable effect sizes to prior work showing moderate effects of knowledge (e.g., $d=.33$; Newberry \& Bailey, 2019) and large effects of expertise (e.g., $\eta^{2}=.26$; Levine et al., 2017). Further, segmentation ability predicted recognition in the current study, which replicated previous work showing better segmentation agreement was related to better memory (e.g., Bailey et al., 2013; Flores et al., 2017; Sargent et al., 2013) and generally supports the fourth principle of the event horizon model. However, this relationship was only present when experts lacked knowledge for the activity, suggesting that experts' superior memory in their knowledge domain was not due to better segmentation.

One explanation is that segmentation helps organize and integrate incoming information during encoding, but semantic knowledge structures (when available) may influence retrieval more so than episodic memory representations created during segmentation. However, when knowledge for the activity does not exist or is impoverished, people have no other option than to rely upon those episodic memory representations to guide retrieval. The current results are not the first to show such an effect. Smith et al. (2020) manipulated participants' familiarity with various everyday activities and found that segmentation ability only predicted memory accuracy in unfamiliar activities 
for both young and older adults. This may suggest that both segmentation and knowledge influence memory, but they do so independently of one another. We want to be cautious when making such claims, though, because we only found this effect in our expert group (segmentation predicted memory in the Overwatch videos) and not in our novice group that lacked knowledge for both types of activity. Additionally, segmentation is not the only encoding mechanism that might benefit memory. Basketball experts in the current study could have engaged in other encoding mechanisms (e.g., semantic chunking or elaboration) to guide their encoding and retrieval of the basketball events. Future research should attempt to tease apart experts' and novices' reliance on schema and event structure when remembering information from events within and outside of one's knowledge area.

Another important note is that the measure of memory in the current study was recognition. Previous work investigating the relationship between segmentation and memory has used recall measures (e.g., Flores et al., 2017; Sargent et al., 2013). It is possible that effects of knowledge on segmentation and memory may be more prominent through recall. Recognition is easier than recall because it provides relevant cues and allows people to rely on retrieval and feelings of familiarity (e.g., Graesser \& Nakamura, 1982; Schwartz, 2018). Recall, on the other hand, does not use (or uses limited) cues, and requires that the person retrieve the information rather than identify the information. Here, the memory effects may not have been large enough to see a benefit of knowledge on segmentation predicting memory due to the availability of cues. We did not use recall in the current study, because control novices could be disadvantaged by not knowing the terminology to describe what they had viewed.

\section{Limitations}

The current study was subject to some limitations. First, recruitment of Overwatch experts was difficult, even after targeting Overwatch players. Part of this difficulty may have been due to unfortunate timing with the release of a new, more popular video game, Fortnite (Ranker, 2018). Future work may have more success by recruiting videogame experts online through sites such as Amazon Mechanical Turk. Moreover, future work should focus on replicating these expertise effects in additional domains of expertise. Previous studies focused on dance and figure skating, which share several characteristics such as learning choreographed movements and often rely on the individual's skills. For the current study, we selected activities that involved a different skill set; however, both activities were team-based and involved multiple players. Thus, given the limited number of domains tested to date, future work should focus on a wider array of domains.
That being said, it should be noted that the merit of the current work remains significant. Both between-subjects and within-subjects comparisons were conducted, which sets this work apart from previous work (e.g., Bläsing, 2015; Levine et al., 2017), and importantly, the control novices did not show the same segmentation benefit as the experts for basketball, suggesting that the effects shown were due to differences in knowledge, not the stimuli.

Second, event memory was only assessed using one memory task. Unfortunately, the order memory results were not interpretable due to extremely low reliability (alpha $=.22$ ). Further, recall was not assessed because of possible differences in vocabulary that could have put control novices at a disadvantage when trying to describe the events of basketball and Overwatch. However, given that recall may be more sensitive to influences of knowledge (e.g., Anderson \& Pichert, 1978; Bransford \& Johnson, 1972), future studies ought to consider including a recall measure despite the vocabulary limitation.

\section{Conclusions}

Ultimately, support for the Event Horizon Model and EST were found, suggesting that knowledge aids memory and that knowledge influences segmentation ability. The current study found that expertise did influence event segmentation ability, but experts' superior memory for events within their field of expertise was not due to better segmentation ability. Evidence was present for both encoding and retrieval differences between experts and control novices; however, preliminary evidence suggests that segmentation and knowledge appear to influence memory independently of one another.

Supplementary Information The online version contains supplementary material available at https://doi.org/10.3758/s13421-020-01118-1

Acknowledgments A sincere thank you to everyone who has been involved with this project: Dr. Heather Bailey, Dr. Lester Loschky, and Dr. Joseph Magliano, for their tremendous guidance and constructive feedback throughout the entirety of this project; Daniel Feller and Jordann Brandner for help with stimuli development; Maverick Smith, for his help with statistical analyses; Destiny Bell and Dr. Barbara Pitts, for their general feedback and support; Jennica Rogers and our team of undergraduate research collaborators, including Jaydan Bruna, Allison Griffin, Marissa Muto, Sydnee Pachek, Nicholas Parker, and Rebecca Ryan, for their help with data collection, data scoring, data entry, and general feedback.

\section{Open practices statement}

The surveys used in this study are provided in the Appendix. The data are currently available through email with the authors, but may be made available after publication through Open Science Framework (https:// osf.io/a86hz/?view only=1411cb0d128146c697f912de198162e5). This experiment was not preregistered.

Author contribution K. Newberry and H. Bailey contributed to the study concept and study design. D. Feller contributed survey materials and 
references. K. Newberry took lead on program development, data collection, and data scoring. K. Newberry also drafted the manuscript, while D. Feller and H. Bailey provided revisions. All authors approved the final version of the manuscript.

\section{References}

Aglioti, S. M., Cesari, P., Romani, M., \& Urgesi, C. (2008). Action anticipation and motor resonance in elite basketball players. Nature Neuroscience, 11(9), 1109.

Allard, F., Graham, S., \& Paarsalu, M. E. (1980). Perception in sport: Basketball. Journal of Sport Psychology, 2(1), 14-21.

Allard, F., \& Starkes, J. L. (1991). Motor-skill experts in sports, dance, and other domains. In K. A. Ericsson \& J. Smith (Eds.), Toward a general theory of expertise: Prospects and limits (pp. 126-152). Cambridge, UK: Cambridge University Press.

Ambrosini, E., Reddy, V., de Looper, A., Costantini, M., Lopez, B., \& Sinigaglia, C. (2013). Looking ahead: Anticipatory gaze and motor ability in infancy. PLOS ONE, 8, e67916. https://doi.org/10.1371/ journal.pone.0067916

Anderson, R. C., \& Pichert, J. W. (1978). Recall of previously unrecallable information following a shift in perspective. Journal of Verbal Learning and Verbal Behavior, 17(1), 1-12.

Bailey, H. R., Zacks, J. M., Hambrick, D. Z., Zacks, R. T., Head, D., Kurby, C. A., \& Sargent, J. Q. (2013). Medial temporal lobe volume predicts elders' everyday memory. Psychological Science, 24(7), $1113-1122$

Baldwin, D. A., Baird, J. A., Saylor, M. M., \& Clark, M. A. (2001). Infants parse dynamic action. Child Development, 72(3), 708-717.

Bartlett, F. C. (1932). Remembering: An experimental and social study. Cambridge, UK: Cambridge University.

Bläsing, B. E. (2015). Segmentation of dance movement: Effects of expertise, visual familiarity, motor experience and music. Frontiers in Psychology, 5, 1500.

Bower, G. H., Black, J. B., \& Turner, T. J. (1979). Scripts in memory for text. Cognitive Psychology, 11(2), 177-220.

Bransford, J. D., \& Johnson, M. K. (1972). Contextual prerequisites for understanding: Some investigations of comprehension and recall. Journal of Verbal Learning and Verbal Behavior, 11(6), 717-726.

Chase, W. G., \& Simon, H. A. (1973). Perception in chess. Cognitive Psychology, 4(1), 55-81.

Chiesi, H. L., Spilich, G. J., \& Voss, J. F. (1979). Acquisition of domain-related information in relation to high and low domain knowledge. Journal of Verbal Learning and Verbal Behavior, 18(3), 257-273.

Clewett, D., \& Davachi, L. (2017). The ebb and flow of experience determines the temporal structure of memory. Current Opinion in Behavioral Sciences, 17, 186-193.

DuBrow, S., \& Davachi, L. (2014). Temporal memory is shaped by encoding stability and intervening item reactivation. Journal of Neuroscience, 34(42), 13998-14005.

Engle, R. W., \& Bukstel, L. (1978). Memory processes among bridge players of differing expertise. The American Journal of Psychology, 91(4), 673-689. doi:https://doi.org/10.2307/1421515

Ericsson, K. A., \& Smith, J. (Eds.). (1991). Toward a general theory of expertise: Prospects and limits. Cambridge, UK: Cambridge University Press.

Feller, D. P., Schwan, S., Wiemer, K., \& Magliano, J. P. (2018). Event segmentation and expertise. Poster presented at the 59th Annual Meeting of the Psychonomic Society, New Orleans, LA.

Flores, S., Bailey, H. R., Eisenberg, M. L., \& Zacks, J. M. (2017). Event segmentation improves event memory up to one month later.
Journal of Experimental Psychology: Learning, Memory, and Cognition, 43, 1183-1202.

French, K. E., \& Thomas, J. R. (1987). The relation of knowledge development to children's basketball performance. Journal of Sport Psychology, 9(1), 15-32.

Furley, P., \& Wood, G. (2016). Working memory, attentional control, and expertise in sports: A review of current literature and directions for future research. Journal of Applied Research in Memory and Cognition, 5(4), 415-425.

Gernsbacher, M. A. (1991). Cognitive processes and mechanisms in language comprehension: The structure building framework. In G. H. Bower (Ed.), Psychology of learning and motivation (Vol. 27, pp. 217-263). Cambridge, MA: Academic Press.

Gilhooly, K. J., Wood, M., Kinnear, P. R., \& Green, C. (1988). Skill in map reading and memory for maps. The Quarterly Journal of Experimental Psychology, 40(1), 87-107.

Goldstone, R. L. (1998). Perceptual learning. Annual Review of Psychology, 49(1), 585-612.

Graesser, A. C., \& Nakamura, G. V. (1982). The impact of a schema on comprehension and memory. Psychology of Learning and Motivation, 16, 59-109.

Hard, B. M., Lozano, S. C., \& Tversky, B. (2006a). Hierarchical encoding of behavior: Translating perception into action. Journal of Experimental Psychology: General, 135(4), 588.

Hard, B. M., Tversky, B., \& Lang, D. S. (2006b). Making sense of abstract events: Building event schemas. Memory \& Cognition, 34(6), 1221-1235.

Hard, B. M., Recchia, G., \& Tversky, B. (2011). The shape of action. Journal of Experimental Psychology, 140, 586-604. doi:https://doi. org/10.1037/a0024310

Hasher, L., \& Griffin, M. (1978). Reconstructive and reproductive processes in memory. Journal of Experimental Psychology: Human Learning and Memory, 4(4), 318.

Herzmann, G., \& Curran, T. (2011). Experts' memory: An ERP study of perceptual expertise effects on encoding and recognition. Memory \& Cognition, 39(3), 412-432.

Huff, M., Papenmeier, F., Maurer, A. E., Meitz, T. G., Garsoffky, B., \& Schwan, S. (2017). Fandom biases retrospective judgments not perception. Scientific Reports, 7, 43083.

Kanakogi, Y., \& Itakura, S. (2011). Developmental correspondence between action prediction and motor ability in early infancy. Nature Communications, 2, 341. https://doi.org/10.1038/ncomms1342

Kurby, C. A., \& Zacks, J. M. (2008). Segmentation in the perception and memory of events. Trends in Cognitive Sciences, 12(2), 72-79.

Kurby, C. A., \& Zacks, J. M. (2011). Age differences in the perception of hierarchical structure in events. Memory \& Cognition, 39(1), 75-91.

Levine, D., Hirsh-Pasek, K., Pace, A., \& Michnick Golinkoff, R. (2017). A goal bias in action: The boundaries adults perceive in events align with sites of actor intent. Journal of Experimental Psychology: Learning, Memory, and Cognition, 43(6), 916.

Loschky, L. C., Larson, A. M., Magliano, J. P., \& Smith, T. J. (2015). What would Jaws do? The tyranny of film and the relationship between gaze and higher-level narrative film comprehension. PLOS ONE, 10(11), e 0142474.

Magliano, J. P., Miller, J., \& Zwaan, R. A. (2001). Indexing space and time in film understanding. Applied Cognitive Psychology, 15(5), $533-545$.

Magliano, J. P., \& Zacks, J. M. (2011). The impact of continuity editing in narrative film on event segmentation. Cognitive Science, 35(8), $1489-1517$.

McGatlin, K. C., Newberry, K. M., \& Bailey, H. R. (2018). Temporal chunking makes life's events more memorable. Open Psychology, l(1), 94-105.

Meinz, E. J., \& Salthouse, T. A. (1998). The effects of age and experience on memory for visually presented music. The Journals of 
Gerontology Series B: Psychological Sciences and Social Sciences, 53(1), P60-P69.

Möller, C., Zimmer, H. D., \& Aschersleben, G. (2015). Effects of shortterm experience on anticipatory eye movements during action observation. Experimental Brain Research, 233(1), 69-77.

Newberry, K., \& Bailey, H. (2019). Does semantic knowledge influence event segmentation and recall. Memory and Cognition, 47, 11731187.

Newtson, D. (1973). Attribution and the unit of perception of ongoing behavior. Journal of Personality and Social Psychology, 28(1), 28.

Newtson, D., Engquist, D., \& Bois, J. (1977). The objective basis of behavior unit. Journal of Personality and Social Psychology, $35(12), 847-862$.

Piras, A., Lobietti, R., \& Squatrito, S. (2010). A study of saccadic eye movement dynamics in volleyball: comparison between athletes and non-athletes. Journal of Sports Medicine and Physical Fitness, $50(1), 99$.

Radvansky, G. A. (2012). Across the event horizon. Current Directions in Psychological Science, 21(4), 269-272.

Radvansky, G. A., \& Zacks, J. M. (2014). Event cognition. Oxford, UK: Oxford University Press.

Radvansky, G. A., \& Zacks, J. M. (2017). Event boundaries in memory and cognition. Current Opinion in Behavioral Sciences, 17, 133140

Ranker. (2018). Most popular video games. Retrieved from https://www. ranker.com/list/most-popular-video-games-today/ranker-games

Rawson, K. A., \& Van Overschelde, J. P. (2008). How does knowledge promote memory? The distinctiveness theory of skilled memory. Journal of Memory and Language, 58(3), 646-668.

Rescorla, R A., \& Wagner, A. R. (1972). A theory of Pavlovian conditioning: Variations in the effectiveness of reinforcement and nonreinforcement. In A. H. Black \& W. F. Proktsy (Eds.), Classical conditioning 1: Current research and theory (pp. 64 99). New York, NY: Appleton-Century-Crofts.

Reynolds, J. R., Zacks, J. M., \& Braver, T. S. (2007). A computational model of event segmentation from perceptual prediction. Cognitive Science, 31(4), 613-643.

Sargent, J. Q., Zacks, J. M., Hambrick, D. Z., Zacks, R. T., Kurby, C. A., Bailey, H. R., . . . Beck, T. M. (2013). Event segmentation ability uniquely predicts event memory. Cognition, 129(2), 241-255.

Schank, R. C., \& Abelson, R. P. (1977). Scripts, plans, goals, and understanding: An inquiry into human knowledge structures. Hillsdale, NJ: Erlbaum.

Schwartz, B. L. (2018). Memory: Foundations and applications. Thousand Oaks, CA: SAGE Publications.

Smith, M., Newberry, K., \& Bailey, H. (2020). Differential effects of knowledge and aging on the encoding and retrieval of everyday activities. Cognition, 196, 104159.

Smith, T. J., \& Henderson, J. M. (2008). Edit blindness: The relationship between attention and global change blindness in dynamic scenes. Journal of Movement and Research, 2(2), 1-17. https://doi.org/10. 16910/jemr.2.2.6

Sommerville, J. A., Woodward, A. L., \& Needham, A. (2005). Action experience alters 3-month-old infants' perception of others' actions. Cognition, 96(1), B1-B11.
Speer, N. K., Swallow, K. M., \& Zacks, J. M. (2003). Activation of human motion processing areas during event perception. Cognitive, Affective, \& Behavioral Neuroscience, 3(4), 335-345.

Swallow, K. M., Kemp, J. T., \& Simsek, A. C. (2018). The role of perspective in event segmentation. Cognition, 177, 249-262.

Tversky, B., Zacks, J. M., \& Martin, B. (2008). The structure of experience. In T. F. Shipley \& J. M. Zacks (Eds.), Understanding events: From perception to action (pp. 436-464). Oxford: Oxford University Press. https://doi.org/10.1093/acprof:oso/ 9780195188370.003 .0019

Vicente, K. J., \& Wang, J. H. (1998). An ecological theory of expertise effects in memory recall. Psychological Review, 105(1), 33.

Wetzels, R., \& Wagenmakers, E. (2012). A default Bayesian hypothesis test for correlations and partial correlations. Psychonomic Bulletin \& Review, 19(6), 1057-1064.

Wilder, D. A. (1978a, 1978b). Predictability of behaviors, goals, and unit of perception. Personality and Social Psychology Bulletin, 4(4), 604-607.

Wöllner, C., \& Cañal-Bruland, R. (2010). Keeping an eye on the violinist: motor experts show superior timing consistency in a visual perception task. Psychological Research, 74(6), 579-585.

Zacks, J. M. (2004). Using movement and intentions to understand simple events. Cognitive Science, 28(6), 979-1008.

Zacks, J. M., \& Swallow, K. M. (2007). Event segmentation. Current Directions in Psychological Science, 16, 80-84.

Zacks, J. M., \& Tversky, B. (2003). Structuring information interfaces for procedural learning. Journal of Experimental Psychology: Applied, 9, 88-100.

Zacks, J. M., Tversky, B., \& Iyer, G. (2001a). Perceiving, remembering, and communicating structure in events. Journal of Experimental Psychology: General, 130(1), 29.

Zacks, J. M., Braver, T. S., Sheridan, M. A., Donaldson, D. I., Snyder, A. Z., Ollinger, J. M., .... Raichle, M. E. (2001b). Human brain activity time-locked to perceptual event boundaries. Nature Neuroscience, 4, 651-655.

Zacks, J. M., Speer, N. K., \& Reynolds, J. R. (2009). Segmentation in reading and film comprehension. Journal of Experimental Psychology: General, 138(2), 307.

Zacks, J. M., Speer, N. K., Swallow, K. M., Braver, T. S., \& Reynolds, J. R. (2007). Event perception: A mind-brain perspective. Psychological Bulletin, 133(2), 273.

Zacks, J. M., Speer, N. K., Vettel, J. M., \& Jacoby, L. L. (2006). Event understanding and memory in healthy aging and dementia of the Alzheimer type. Psychology and Aging, 21(3), 466.

Zacks, J. M., Kurby, C. A., Eisenberg, M. L., \& Haroutunian, N. (2011). Prediction error associated with the perceptual segmentation of naturalistic events. Journal of Cognitive Neuroscience, 23(12), 40574066.

Publisher's note Springer Nature remains neutral with regard to jurisdictional claims in published maps and institutional affiliations. 